\title{
The Application of Metaphor in College English Vocabulary Teaching
}

\author{
Wang Xiaoge ${ }^{1, \mathrm{a}}$, Lv Ling $^{1, \mathrm{~b}}$ \\ ${ }^{1}$ Qingdao Huanghai University \\ Qingdao, China \\ a86284769@qq.com, b2381261329@qq.com
}

\begin{abstract}
English is a language which is quite different from Chinese. It has many characteristics of its own, and has its regional, historical and cultural differences. For Chinese students who lack English learning environment, memorizing English words is a difficult problem in English learning. Learning English words requires a lot of time and effort, and also must master some scientific methods. As important human cognition, metaphor plays an important role in vocabulary acquisition. The related theory of metaphor is introduced into the teaching of college English vocabulary, it can make students' vocabulary learning more consistent with the human cognitive law, to help student master more effective vocabulary learning methods, in order to improve and reform the quality of college English vocabulary teaching.
\end{abstract}

Keywords-Metaphor; College English teaching; Vocabulary teaching; Reform

\section{INTRODUCTION}

Vocabulary acquisition is a difficult process involving semantic, syntactic, lexical, and phonetic and structures. The traditional college English vocabulary teaching is affected by structuralism, There is no intrinsic connection between words (Signifier or speech image) and meaning (Signified or concepts), teaching words as an isolated unit, the phenomenon that causes students to recognize a large number of words and not apply them, it's putting a lot of pressure on students, thus losing the interest and initiative in learning English [1]. As an important cognitive method of human beings, metaphor plays an important part in the formation of human cognition and its conceptual structure. Shu Dingfang Pointed out: "Metaphor theory has positive guidance and application value to language teaching. Language teachers can use metaphor theory to explain the change of language meaning, explain the interrelationship between lexical meanings; at the same time, the conceptual metaphor theory can be used to explain the systematic and interrelationship between different forms of metaphor in language". Therefore, introducing metaphorical cognitive theory into college English vocabulary teaching will surely infuse vocabulary teaching with new vitality and provide new ideas.

\section{RELATED THEORIES AND RESEARCH}

Metaphor is a universal phenomenon in which people use metaphors in large quantities all the time. Metaphor is not only found in various literary works, but also in politics, science and technology, economy, religion and other fields. Most of our experience and activity are metaphorical in nature. Most conceptual systems are constructed by metaphors. Due to the limitation and narrowness of thinking, many things people need to understand and express in real life, especially in an abstract field, which has to be accomplished through the medium of metaphor. No matter which English dictionary, there will always be a list of words and meanings listed below. The formation of metaphor has created symbolic ambiguity in natural language. In the daily use of language, metaphor leads to the word polysemy and language novelty, and metaphor creates new language.

\section{A. Meanings of metaphor}

With the development of the study of metaphor, people's understanding of metaphor breaks through the limitation of traditional rhetoric, that metaphor is not only a linguistic phenomenon, but also a phenomenon of human cognition. Richards pointed out that metaphor is the ubiquitous principle of human language. The publication of Johnson's Metaphors has led to a basic consensus on the fact that "metaphor is everywhere", it also recognizes that metaphor is a question of thinking, a fixed and stable projection between two conceptual domains, and argues that metaphor is embedded in language and culture, influence and restrict people's mind. Since then, the research on the cognitive function of metaphor has gradually developed [2].

The research on the cognitive function of metaphor in foreign countries has been expanded into many fields. In contrast, in the field of foreign language teaching, there is much less applied research on the cognitive function of metaphor, and there are many theoretical studies and less empirical studies. Danesi first introduced the metaphor ability into the field of second language acquisition, and believed that the metaphor ability is the substructure of the concept fluency, which is the ability to recognize and use the new metaphor in the process of speech and writing. At present, one of the few empirical studies of basic consistent conclusion, there is a significant difference between the two learners' metaphorical 
abilities, generally speaking, the ability of metaphor comprehension is not strong, which requires a lot of hard work and the guidance of teachers.

\section{B. The development of metaphor}

Domestic metaphor research began in the 1990s. Yan Shiqing, $\mathrm{Hu}$ Zhuanglin and others have published related monographs, from the perspective of cognition. It is proposed that metaphor is a kind of thinking paradigm. Metaphor ability and logical ability are the basic thinking ability of human beings, and the mental activity of human life is diffused throughout life. Huang explored the metaphorical awareness and foreign language vocabulary teaching [3]. Although these research gives great affirmation to the importance of metaphorical abilities, the application of these researches in practical teaching is very rare.

This paper mainly discusses the influence of metaphor on vocabulary acquisition and its application in College English vocabulary teaching.

\section{THE INFLUENCE OF METAPHOR ON VOCABULARY ACQUISITION}

As a whole, human language is a metaphorical symbolic system. Depending on statistics, $70 \%$ of the vocabulary in English are metaphorical or metaphorical [4]. Linguists think that the process of lexical cognition is a complicated process, which is composed of many related parts, rather than confined to the form and sense of vocabulary, the extension of the word's metaphorical meaning is an integral part of the vocabulary cognition; moreover, the concept metaphor is fully utilized in terms of lexical input, deep processing of vocabulary and vocabulary output. Craik and Lockhart noted that in the process of vocabulary cognitive vocabulary for deep processing can promote the meaningful understanding of the vocabulary and produce long-term memory, only by understanding the metaphorical meaning of words can we achieve the purpose of deep processing of words.

\section{A. Content of vocabulary teaching}

Vocabulary output usually includes three aspects of output. Reproduction, perception and creative use. The imaginative use of vocabulary is the process of mapping from a specific conceptual domain to an abstract conceptual domain. From this, we can note that there is a close relationship between lexical cognition theory and conceptual metaphor, metaphor plays a decisive role in lexical cognition and can promote understanding, memory and innovation of lexicon. Traditional vocabulary teaching often ignores the metaphorical meaning and connotative meaning of vocabulary. This is largely limited the learners on depth of vocabulary cognition, so that students cannot grasp the different meanings of core words in different contexts and the powerful expression of polysemy. The real acquisition of a vocabulary not only to understand its meaning, but also need to understand the other meaning, from it can be derived from the word, it have what connection with other entries in the dictionary, its syntactic function and its use range in different situations and functions and the difference between synonyms.

\section{B. Metaphor application of vocabulary teaching}

As an often neglected link and a significant resource of polysemy, metaphor is the main motivation of the word meaning, the mechanism of expression, the source of synonym and polysemy, the release of strong feelings, the way to fill the lexical gap (Ullmann1962). In addition, the generation of metaphor relies on background and empirical knowledge, and it can also trigger and express this knowledge, so metaphor can promote the understanding of vocabulary. Therefore, metaphor should be regarded as an important guiding method and main content of college English vocabulary teaching, enabling students to deepen the language learning to the depth of cognitive thinking, to understand the metaphorical link between English vocabulary and thinking, so that we can remember, consolidate and use vocabulary from the cognitive point of view in the usual vocabulary learning.

\section{THE APPLICATION OF METAPHOR IN COLLEGE ENGLISH VOCABULARY TEACHING}

\section{A. Pay attention to vocabulary learning in basic category}

The basic category is the most elementary psychological level to distinguish things from human beings. It is important base point and reference point of cognition. The basic category vocabulary refers to the simple, less syllable, analytical negatively and easily acquired native words [5]. It is the most direct and uncomplicated level of the interaction between people and the world. When a lack of corresponding vocabulary concept exists the expression language daily, people can't create new words only, but transfer their thinking from the known cognitive domain to the unknown things and abstract concept of the cognitive domain with the influence of metaphorical thinking. They use creative metaphorical thinking to recognize and name new things, to express abstract concepts, that is, to replace words that describe the same shape or function from the existing undeveloped category words.

As a result, the basic category words have more metaphorical use than other category words, and have a higher frequency of use. And it can be said that the basic category words constitute the basic meaning of the basic single word and words, and they are the most commonly used words and the most elementary meaning. Therefore, teachers and students should pay more attention to the study of basic vocabulary in College English vocabulary teaching. When teaching, teachers should not only guide students to find out the basic meaning of the basic vocabulary, but also take the teaching of basic vocabulary metaphorical meaning as an organic part of English Vocabulary Teaching. What's more, teachers should combine the operation mechanism of metaphor with the associative cognition of lexical semantics, and pay attention to the repetition of lexical meaning, so as to arouse the students' thinking of metaphor, form a semantic network in the mind, and ultimately improve the students' ability of conceptual fluency. 


\section{B. Deep connection among the polysemy senses}

The polysemy of words in English is expected to be very common. It is the purpose requirement of the principle of linguistic economy and the principle of cognitive economy. The meanings and use of polysemy words from a category of meaning. There are certain connections between the senses of polysemy words. The most basic is the central senses. Other senses are produced by the development of the inner senses through metaphor. Some of them have become the literal meaning of the word and are not seen by people. The connection between multiple meanings of polysemy words is not arbitrary, but develops from a typical model through a precise semantic extension mechanism. And each link between the values is rational. Metaphor takes advantage of the similarity between one thing and another to transfer the structure and characteristics of the source concept domain to the target domain, use the source concept involving domain words to refer to and describe the target domain, and it makes old-meaning words generate new meaning based on both the differences and relationship between the new meanings, so polysemy words are created. To clarify the cognitive mode of the native language hidden behind the polysemy, and to understand the connection patterns among the senses of polysemy words will undoubtedly help English learners understand and memorize the basic vocabulary, phrases and vocabulary to deepen understanding, enhance learning ability and memory of words in practice and flexible the use of vocabulary. In vocabulary teaching, teachers should guide students to follow the rules of metaphorical thinking, associative vocabulary meaning, derive its various meanings according to the cognitive model by vocabulary meaning. When explaining the various meanings, teachers can associate its original meaning with every layers of metaphoric meaning, and guide students to know the importance of metaphorical thinking rules of language in the process of deriving from a layer of meaning so as to guide students to follow the rules, ratiocinate step by step, to get more meaning eventually.

\section{Differences of vocabulary metaphor}

Cognitive linguistics holds that culture does not exist somewhere outside the language, but in every aspect of the language itself, exist in every word, in each grammatical construction, and it can be found through linguistic behavior and linguistic analysis, and constantly communicated by language. Learners should feel the close connection between language and culture in the process of language learning. In other words, the process of language learning is not only the process of learning language itself, but also the process of learning language knowledge and cultural knowledge. Furthermore, understanding the mutual contact among the language mechanism, meaning generation, language and culture, and the concept will not only contribute to better and more in-depth understanding of the target language and the target language culture, but also a deeper understanding of Chinese language and Chinese culture, so the new understanding of the world will be formed. Metaphor is a concentrated expression of culture in language, a combination of language and culture, and many contents of culture need to be expressed and inherited through metaphor and the culture of the language can be examined with that. Therefore, teachers should help students realize the cultural and cognitive features of vocabulary in the process of learning each word, and can't understand and indicate the corresponding language metaphor in the target language by conceptual metaphor in the mother tongue only. Through the process of vocabulary learning, learners will gradually find the manipulation function of metaphor, and realize how flexible and powerful the language is, and fully understand the cultural knowledge, mode of thinking and characteristics of language infiltration.

\section{Metaphorical thinking}

Metaphor is a systematic way for people to think, act and express ideas, and the whole mapping across conceptual domains, and metaphorical expression is only the outward representation of mapping across conceptual domains. People are still familiar with the specific concepts they know, concrete concepts, unfamiliar concepts and abstract concepts. In terms of expression, people have a tendency to talk about another concept by talking about each aspect of a concept. November believes that the new words presented together are easy to remember and learn more than the isolation of them in the same category, because it allows learners to form new neural connections and more effective organizational knowledge. If learners are encouraged to categorize their vocabulary according to certain metaphorical patterns, it must be useful to enlarge their vocabulary. When students are learning and memorizing new words, they can find the connection between the new words and the old, the abstract words and the concrete by means of the metaphorical mode of thinking, so that the words can be stored for a long time and are not easy to be forgotten. For example, affixes and compound words in English belong to metaphorical word formation, and they can memorize more words through the meaningful association of metaphor. In addition, understanding the role of metaphorical thinking in the creation of new words and meanings and attaching importance to learning basic vocabulary, can help students deepen their impression and strengthen memory from the cognitive perspective, and learning to associate boldly with the metaphor of thinking will make the vocabulary learning no longer boring, but more meaningful.

\section{GRASP THE BASIC MEANING OF VOCABULARY THROUGH CONCEPTUAL METAPHOR}

The English vocabulary includes two meanings, the basic meaning and meaning of the extension. The rise and application of metaphor make the word meaning more abundant and multi-layered, which appeared in polysemy situation. In the meaning of vocabulary, the basic meaning is the basic part of the lexical meaning. Other meanings are derived indirectly through the application of the metaphorical way, and the implied meanings cannot exist without the basic meaning. Therefore, in the teaching of English vocabulary, teachers should make students clear the meaning and value of the basic meaning of English vocabulary, and then guide students to find more meaning behind the basic meaning, so that students more comprehensive understanding of the English vocabulary, thereby enhancing students' efficiency in learning English vocabulary. 


\section{A. The systematicness and cognition of metaphor}

Metaphor embodies the systematicness of thought and language, which enables us to understand and experience another thing or experience with one thing and experience. First of all, metaphor mapping is selective. In the process of mapping the source domain to the target domain, the characteristics of the vehicle are selective. Some features are highlighted or hidden as was required to be mapped onto the target domain to eliminate the understanding of the target domain by other features of the source domain. Secondly, in order to secure the orderly progress of human cognition, each of the metaphorical expressions in a certain systematic way follows a paradigm, which is dominated and restricted by metaphor. Thirdly, the metaphorical structure shows a certain level of hierarchy. This hierarchy structure is associated with each other. The lower one inherit the characteristics of the higher layer mapping.

\section{B. Educational expert opinion}

Metaphor is not only a linguistic rhetorical device, but also a way for people to conceptualize the surrounding world, which has a powerful cognitive function. Lakoff \& Johnson emphasizes: "In the most fundamental sense, mind is based on body experience, meaning is based on body experience, and thinking is based on body experience." We can talk about "mind, meaning and thinking" collectively as cognition, so that "cognition is experiential" and experience is an important fulcrum of human cognition. However, metaphor is based on experience, so the metaphor is undoubtedly cognitive. Metaphor is through familiar area to understand cognitive unfamiliar, this process of understanding is the process by which mankind learns of new things through his own experience, using the understanding of the surrounding known things, and linking two totally unrelated things Cognitive Process. As Hu states that the cognitive process is at the heart of metaphors, which make the familiar and unfamiliar things parallel, deepening our knowledge of unfamiliar things [6]

In English teaching, teachers integrate metaphor into classroom teaching, easy-to-understand concepts and images of everyday life into abstract English phenomena, guided students to make use of their main initiative, so that students can feel better the theory and interest of English learning, so as to help them strengthen their understanding and application of English sentences.

\section{CONCLUSION}

Applying metaphor theory to college English vocabulary teaching, vocabulary learning will no longer be a simple vocabulary memory category, but sublimate to the level of pragmatic and thinking mode. It not only helps learners learn vocabulary deeply, firmly grasp the different meanings of basic core words in different contexts and the strong expressive power words, but also can make learners understand the target language profound national culture characteristic, and gradually expand the perspective of the observation and thinking, to promote rational thinking gradually. In addition, due to the "divergent and jumping nature" of metaphor associations, English vocabulary teaching can become more vivid and interesting and greatly stimulate students' learning motivation.

\section{REFERENCES}

[1] Sun Y M, Gao J M. "Advantages of Ideational Metaphor in College English Writing Teaching". Shandong Foreign Language Teaching Journal, 2011. pp.225-230.

[2] Jun-Xing L I. "The Use of Metaphor in College English Vocabulary Teaching and Learning". Journal of Hubei Radio \& Television University, 2008, pp.383-388.

[3] Huang Y L. "A Cognitive Approach to the Application of Metaphor in College English Textbooks". Journal of Huaihua University, 2008, pp.65-79.

[4] Zheng Q. "The Application of Metaphor Theories in College English Vocabulary Learning”. Overseas English, 2014,pp.110-118.

[5] Sang M L, University J A. "The Teaching of Vocabulary in College English Under the View of Cognitive Metaphor". World \& Chongqing, 2013, pp.1246-1256.

[6] Cai J. "Characteristics and solutions of college English teaching in transition”. Foreign Language Teaching \& Research, 2007, pp.27-32. 